\title{
FROM JABEOŃSKI TO FEMTOSECONDS. EVOLUTION OF MOLECULAR PHOTOPHYSICS
}

\author{
M. KASHA \\ Institute of Molecular Biophysics, Florida State University \\ 451 MBB-4380, Tallahassee FL 32306-4380, U.S.A.
}

\begin{abstract}
A presentation is given, with retrospective commentary, on the experimental and theoretical contributions to key steps in the evolution of the framework of contemporary molecular photophysics from the Jabłonski Diagram to femtosecond range excitation phenomena. The distinctive features of polyatomic molecules separating their behavior from atomic and diatomic molecules are emphasized. Justification is given for the statement that spin-orbital coupling with its relativistic component commonly dominates the molecular excitation dynamics of light-(low-Z)-atom molecules. The paper deals with single-photon, single-molecule excitations. Some examples of single-photon, multi-molecule and multi-photon, single-molecule excitation phenomena are listed. A selection of these is made to illustrate the prevalence of femtosecond excitation modes.
\end{abstract}

PACS numbers: 33.50.-j, 33.50.Dq

\section{Introduction}

Molecular photophysics and its spectroscopic, quantum mechanical, and excitation dynamics framework experienced a remarkable retardation of development compared with the advanced state of understanding of atomic and diatomic systems by 1935. Surveying the origins of this delayed development offers an opportunity of (a) examining the unraveling of the unique complexities of polyatomic systems and (b) highlighting the contrasting and unique behavior of these relative to the simpler behavior of atomic and diatomic cases. This presentation is aimed at offering a commentary on the key phenomena discovered and interpreted over the past six decades since Alexander Jabłoński's first clear step $[1,2]$ in the direction of contemporary molecular photophysics [3].

\section{Photoluminescence of dyes}

Molecular dyes and their prevalent fluorescences dominated the attention of early investigators for decades preceding the period covered by this survey, starting with the Stokes [4] study of the relation of molecular fluorescence to absorption. In retrospect, it is quite clear that this preoccupation with molecular 
dyes and their vivid colors and fluorescences served as a severe handicap to the full understanding of molecular photophysics. This limitation arose from the special properties of dye molecules: (a) high oscillator strength of first absorption band, yielding a correspondingly very short fluorescence mean lifetime (nanoseconds) (via the Einstein absorption and emission coefficient relation); (b) usually a very limited quantum yield of phosphorescence, as a consequence of (a); and (c) a very small singlet-triplet configurational split $\left(\approx 1500-2000 \mathrm{~cm}^{-1}\right)$, resulting in submersion of any phosphorescent band(s) in the long wavelength tail of the fluorescence. Because the observation of a molecular phosphorescence seemed to be associated with the inclusion of the dye molecule in a solid state matrix, the occurrence of this phenomenon itself posed a mystery.

\section{Mystery of molecular phosphorescence}

Before the 1940's, physicists tended to regard the observable phosphorescence of dyes as strictly a solid state phenomenon. Phosphorescence of mineral crystals doped with an impurity was a widely studied phenomenon and the very long-lived phosphorescence observed was interpreted as an electron recombination phenomenon (non-exponential) activated by thermal excitation. This model was suggested by Francis Perrin [5] using exactly the schematic model proposed by Jean Perrin, his father and predecessor, now applied to dye molecule phosphorescence (Fig. 1). This diagram superficially seems to bear a striking parallel to the famous Jabłoński Diagram (Fig. 2), especially in describing the energy level $\alpha$ as the metastable state, which is exactly the designation Jabłonski gave to his state $M$. However, in every aspect, Perrin indicates with complete clarity that his level $\alpha$ represents a metastable trap energy level. His descriptions are vivid: The energy level $\alpha$ can give phosphorescence uniquely by reactivation to the fluorescent state $a$, the phosphorescent spectrum thus must always be identical with the fluorescence; the lifetime of the energy level $\alpha$ would be infinite at the temperature zero $\mathrm{K}$ (no thermal activation); the depth or energy of $\alpha$ is determined by the solid state matrix; $\alpha$ is not related fundamentally to the electronic states of the molecule, and does not exhibit normal transition probability relations between the level $\alpha$ and the ground state $A$ (Fig. 1).

The Jabłonski $[1,2]$ Diagram is considered as the first clear step ahead in the development of molecular photophysics because it identified the critical lowest three states $N, F$, and $M$ as quantized molecular electronic states. Jabłoński then explored the quantum yield and kinetic relations between these states, especially including direct $N \rightarrow M$ electronic absorption (of severely limited intensity), the $M \rightarrow N$ direct phosphorescence, and also the temperature dependent $M \sim F$ reactivation of what is now called a delayed fluorescence $(M) \sim F \rightarrow N$. Jabłoński did not admit the possibility that the state $M$ could be a triplet state (however, see Sec. 9).

A mystery on the origin of the long-lived molecular phosphorescence remained, as revealed by the speculations of researchers who followed Jabłoński. All of these workers were preoccupied with the apparent special role of the solid state environment in which the molecular phosphorescence was observed, and all of these were concerned principally with dye molecules in rigid glass media (frozen 


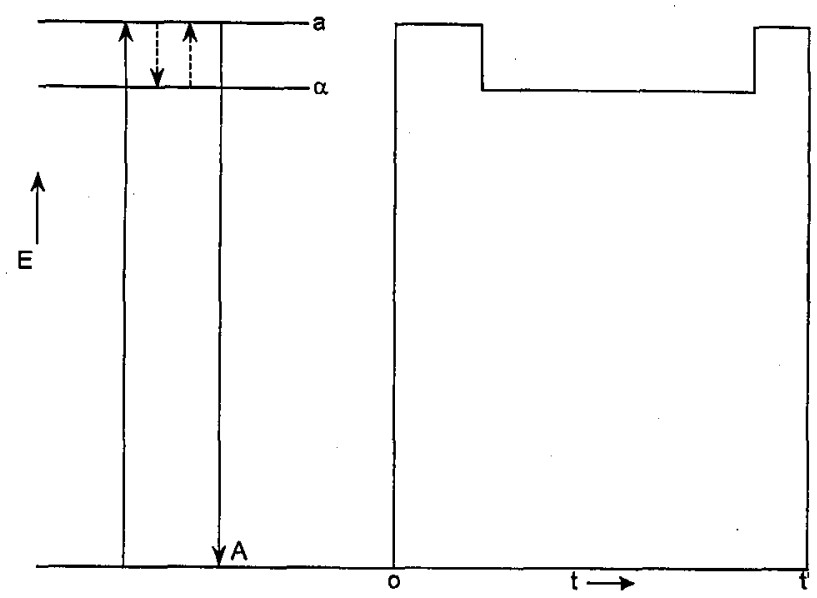

Fig. 1. Francis Perrin Diagram. Metastable trap model.

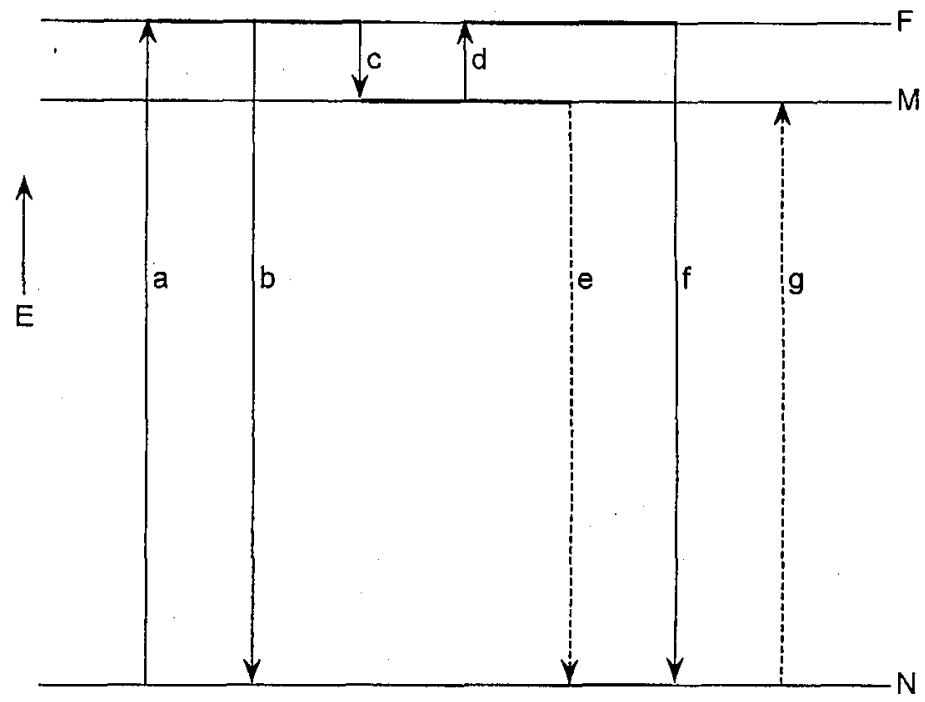

Fig. 2. Jabłoński Diagram. Dye molecule Iuminescence.

organic solvent mixtures, melted sugars such as glucose or dextrose supercooled to a glass, etc.). Thus, Franck and Livingston [6] in 1941 attributed "the phosphorescent state" to a tautomer of the ground state, and also in 1941 Lewis, Lipkin, and Magel [7], introducing the appellation Jabtonski Diagram, considered the metastable state to be a geometrical isomer clamped in the rigid glass matrix; the latter authors also speculated that a triplet state multiplicity might be involved. Terenin [8] in 1943 adopted tautomerism, geometrical isomerism, a triplet state possibility, and also possibly hydrogen bonding to the solvent matrix as a further origin of the metastability. The present author has compared these mechanisms in 
detail [9]. So the mystery remained and was profoundly complicated by the objections of physicists who pointed out the quantum mechanical stricture [10] against ever observing a triplet $\leftrightarrow$ singlet transition in molecules consisting of only the lighter (low atomic number) atoms $\mathrm{C}, \mathrm{N}, \mathrm{O}$, and $\mathrm{H}$.

\section{Triplet-singlet transitions in organic molecules}

In 1944 G.N. Lewis and the author [11] published a paper which explored the wider meaning of the Jabłoński Diagram, by extending the main concepts to polyatomic molecules in general. This had several great advantages. Such non-dye molecules have their principal absorption spectra in the ultraviolet, including the first absorption band. Secondly, with a greater range of spectral space below the $S_{0} \rightarrow S_{1}$ transition, the much larger singlet-triplet configurational splits [12] (arising from the greater electron repulsion in the smaller space of a limited polyatomic, e.g., benzene, compared with the much greater orbital space of the dye molecule) allowed low-lying $T_{1} \rightarrow S_{0}$ transitions to be observed. Additionally, the vibrational fine structure could be resolved and used to further characterize the luminescences. These points are illustrated by Fig. 3 for the phenanthrene luminescence, which exhibits a clearly resolved phosphorescence spectrum, with a singlet-triplet split of $5500 \mathrm{~cm}^{-1}$. Obviously, one principal feature of the Jabloński Diagram is now eliminated for these large $S_{1}-T_{1}$ splits in most organic molecules: the thermal reactivation $T_{1} \leadsto S_{1}$.

From the study of a large number of such polyatomic molecules, we now can generate our Electronic State Diagram as given in Fig. 4. But what permitted this sudden expansion of understanding of the Jabłoński Diagram? The spectroscopist Robert Mulliken had lectured in Berkeley and was suggesting that all

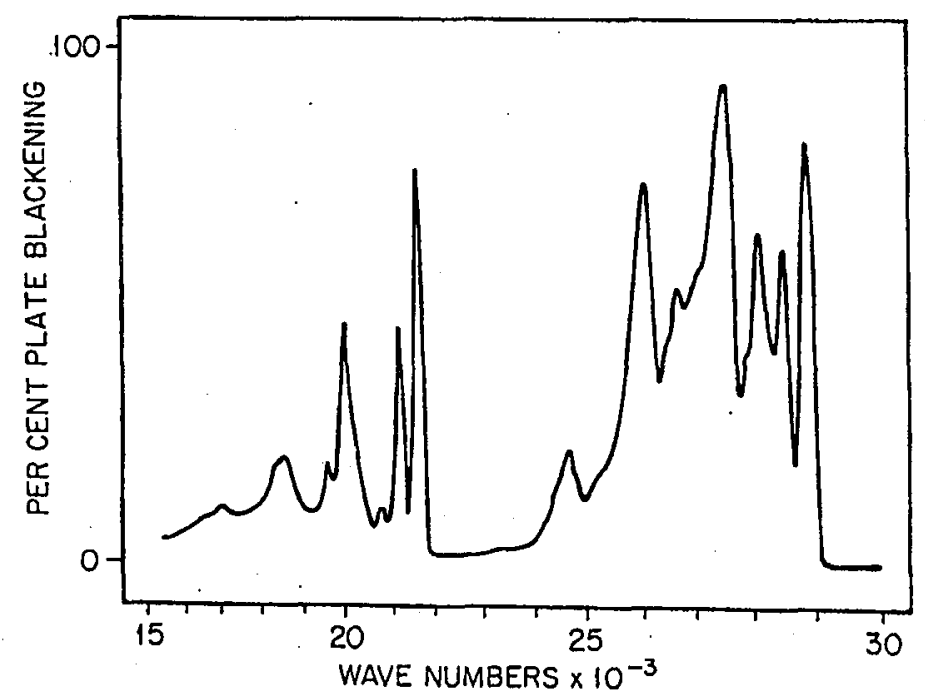

Fig. 3. Total emission spectrum of phenanthrene: $T_{1} \rightarrow S_{0}, S_{1} \rightarrow S_{0}$. 


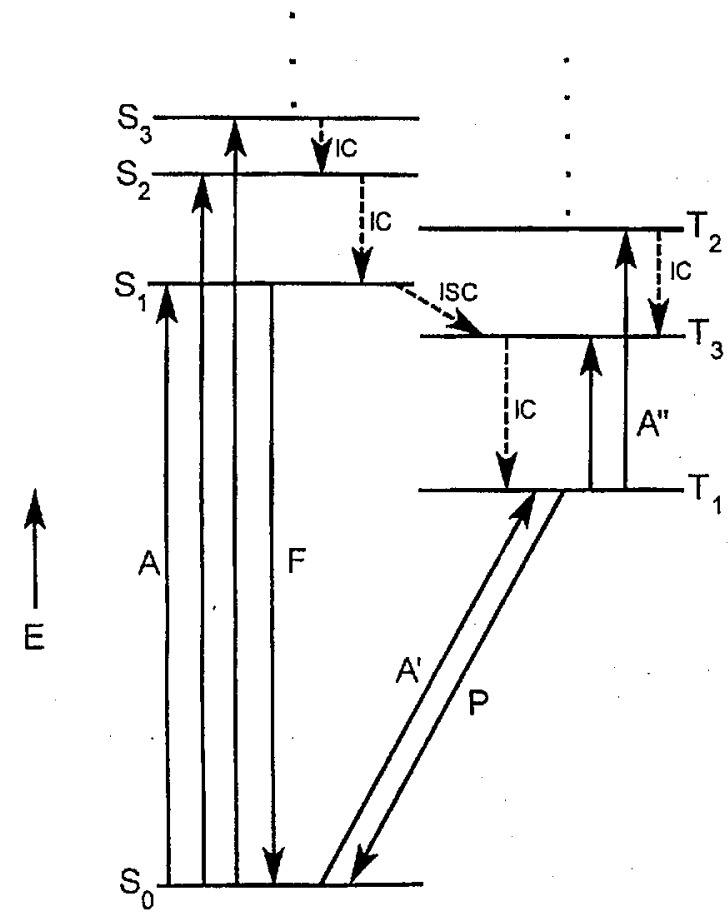

Fig. 4. Electronic State Diagram. Polyatomic molecule excitation.

molecules should give evidence of a triplet state lying below the lowest singlet excited state [13]. But where were these triplet states, and how to find them? With some uncertainty, Lewis already mentioned a triplet possibility in his 1941 paper. But when Lewis saw the many dozens of cases of beautifully-developed phosphorescence spectra in my work on smaller polyatomics, his intuition took a leap and said, "Then Jabłoński's metastable state must be the triplet state that Mulliken is talking about!" So our 1944 paper was titled Phosphorescence and the Triplet State, and presented spectral data on almost one hundred molecules.

We had not proved our thesis, but our researches showed exhaustively that the phosphorescence spectrum was truly an intrinsic molecular phenomenon independent of the rigid glass matrix, and that $S \leftrightarrow T$ transitions could be observed even in fluid media and the gas state [14]. At that time, we had a little knowledge of spin-orbital coupling theory and the quantum mechanical limitations imposed by it. Our thesis met a storm of controversy [9].

\section{Singlet-triplet mixing in polyatomic molecules}

In any complex subject, an advance can be made only by probing the requirements of fundamental theory. In the present case, the quantum mechanical perturbation theory must be applied to understand how a rigorously-restricted electronic spin change involved in the so-called "triplet-singlet transition" could ever take place. Fortunately, in Berkeley I had a magnificent mentor in the theoretical physicist David Bohm, then a member of the Physics Department. $\mathrm{He}$ 
introduced the quantum mechanical theory of spin-orbital perturbation to my seminar group.

The essence of the idea in spin-orbital perturbation lies in the possibility that pure states can gain an admixture of other state components if an appropriate interaction Hamiltonian term can be applied. Thus, an admixed (or impure) state can be composed of two pure states

$$
\psi_{n}^{1}=\psi_{n}^{0}+\sum_{m}^{\prime} \frac{H_{m n}^{\prime}}{E_{m}^{0}-E_{n}^{0}} \psi_{m}^{0}
$$

where the pure states $\psi_{n}^{0}$ and $\psi_{m}^{0}$ can be mixed by the mixing coefficient which contains the matrix elements of the appropriate Hamiltonian $H^{\prime}$ over the states $\psi_{n}^{0}$ and $\psi_{m}^{0}$, and the energy denominator $E_{m}^{0}-E_{n}^{0}$. Applying this first order perturbation formula to the lowest triplet state $T_{1}$, and introducing the spin-orbital Hamiltonian operator (cf. Fig. 5), the question is which of the nearest singlet states $S_{m}$ will give a non-zero integral over $\widehat{H}_{\mathrm{so}}$ ? This question is decided by the symmetry of the states involved.

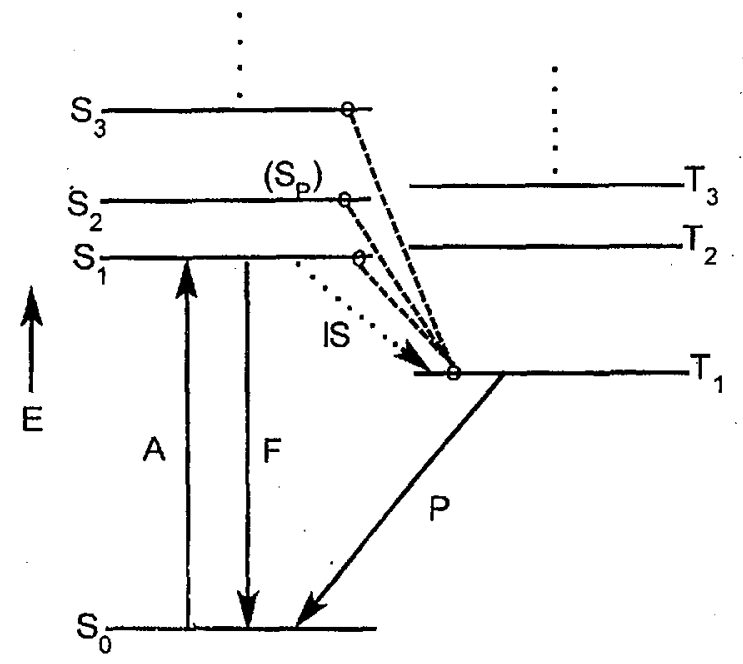

Fig. 5. Singlet admixture in triplet states.

Thus, although the pure spin state $T_{1}^{0} \rightarrow S_{0}^{0}$ transition is rigorously forbidden, the transition $T_{1}^{1} \rightarrow S_{0}^{0}$ can be made permitted to the (limited) degree that the $T_{1}^{1}$ state now contains some singlet state admixture (as the impure triplet state $\left.T_{1}^{1}\right)$.

$$
T_{1}^{1}=T_{1}^{0}+\sum_{m}\left[\frac{\left\langle S_{m}^{0}\left|\hat{H}_{\mathrm{so}}\right| T_{1}^{0}\right\rangle}{E_{m}\left(S_{m}^{0}\right)-E_{1}\left(T_{1}^{0}\right)}\right] S_{m}^{0} .
$$

The rate constant for Einstein emission coefficient $A_{21}$, or $k_{p}=1 / \tau_{p}^{0}$ is then expressible as the square of the electric dipole transition moment 


$$
\frac{1}{\tau_{p}^{0}}=\frac{32 \pi^{3} \bar{\nu}_{T S}^{3}}{3 \hbar c^{3}}\left|\frac{\left\langle S_{p}^{0}\left|\widehat{H}_{\mathrm{so}}\right| T_{1}^{0}\right\rangle}{E_{p}\left(S_{p}^{0}\right)-E_{1}\left(T_{1}^{0}\right)}\left\langle S_{p}^{0}\left|\sum_{i} e_{i} r_{i}\right| S_{0}^{0}\right\rangle\right|^{2}
$$

Thus, the intensity of the $S_{0}^{0} \rightarrow S_{p}^{0}$ transition determines the $T_{1}^{1} \rightarrow S_{0}^{0}$ rate constant. This commonly involves a restriction of about $10^{6}$, so that a $10^{-9} \sec S_{p}^{0}$ (or $S_{1}^{0}$ ) intrinsic mean lifetime would correspond to a $10^{-3}$ sec $T_{1}^{1}$ intrinsic mean lifetime. The summation over mixing states is omitted here for simplicity.

A crucial factor lies in the composition of the spin-orbital operator. Expressed for a single atom with a Coulombic field

$$
\widehat{H}_{\mathrm{so}}=\xi\left(\frac{Z^{4}}{n^{3}}\right) \sum_{i} \ell_{i} \cdot s_{i} .
$$

It is at once apparent that the spin-orbital coupling factor $\xi$ has an enormously high-power dependence on $Z$, the atomic number. The $Z^{4}$ dependence becomes a $Z^{8}$ dependence in the transition moment! This huge dependence is somewhat mitigated by the principle quantum number $\left(n^{3}\right)^{2}$ in the denominator. Learning of this great dependence on $Z$, it was found that the radiationless $S_{1} \leadsto T_{1}$ intersystem crossing $[15,16]$ had the expected sensitivity to $Z$ by studying the $T_{1} \rightarrow S_{0}$ relative yield in $-\mathrm{Cl},-\mathrm{Br},-\mathrm{I}$ substituted aromatics (Fig. 6). McClure then made a parallel study [17] of $T_{1} \rightarrow S_{0}$ lifetime sensitivity to $Z$.

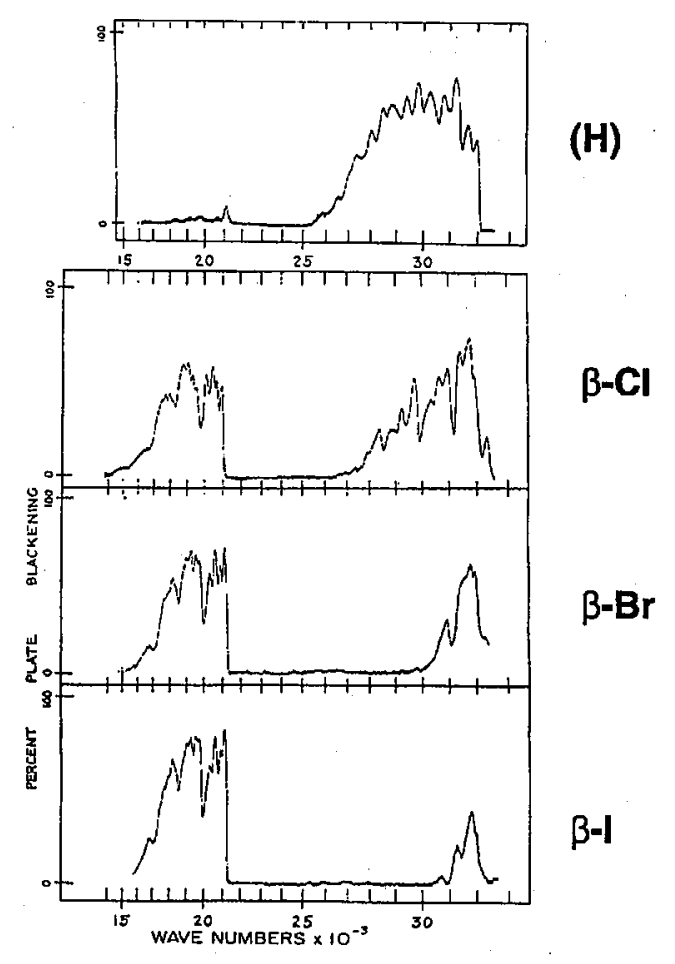

Fig. 6. Heavy atom perturbation of intersystem crossing in naphthalene. 
Intermolecular spin-orbital effects were soon realized. The knowledge that the quenching constants by the Stern-Volmer equation for the ions $\mathrm{Cl}^{-}, \mathrm{Br}^{-}, \mathrm{I}^{-}$, increase rapidly with increasing $Z$ for quenching the fluorescence of dyes in aqueous solution, led to the idea that singlet-triplet absorption in liquid solution could be enormously enhanced as well in heavy-atom-containing solvents, as shown in Fig. 7 [18]. This study was readily extended [19] and the "external heavy atom effect" is now generally used. Induced triplet-singlet emission using I-atom containing media has also been observed, spectacularly in the $800 \mathrm{~nm} T_{1} \rightarrow S_{0}$ case of $\mathrm{C}_{60}$ fullerenes [20]. This necessity of inducing $T_{1} \rightarrow S_{0}$ emission arises especially in cases of very low-lying triplet states approaching or in the near infrared. In such cases, the induced radiative $\mathcal{T}_{1} \rightarrow S_{0}$ emission can compete with the radiationless process, which becomes more probable the closer $T_{1}$ lies to the ground state.

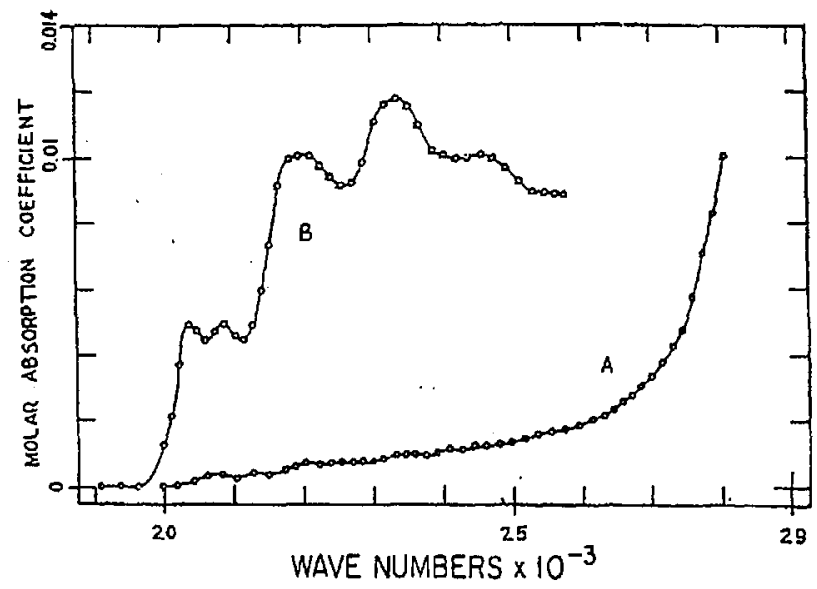

Fig. 7. Solvent-induced singlet-triplet absorption. Alpha-chloronaphthalene in ethyl iodide (1:2 mixture).

Now we must return to the provocative question. Understanding spin-orbital coupling and its tremendous dependence on $Z$, how can we account for the facile observation of $T_{1} \rightarrow S_{0}$ emission in light-(low- $Z$ )-atom molecules containing only $\mathrm{C}, \mathrm{N}, \mathrm{O}$, and $\mathrm{H}$ atoms (atomic number $Z=6,7,8 ; 1$. respectively)?

We shall first examine the various causes of orbital excitation in polyatomic molecules. Then we shall consider the role of dynamics in the excitation of the lowest triplet state.

\section{Characterization of molecular electronic states}

Molecular geometrical structures permit the designation of distinctive orbital types originating from the component atoms, in reference to Cartesian axes. The most common bonding orbital types are $\sigma$ and $\pi$. These designations are derived from atomic orbital angular momentum designations, $\sigma, \pi, \delta, \ldots$ for $\ell$ values $0,1,2, \ldots$ We find it convenient in zeroth order (no mixing) to refer to these orbitals in a Cartesian framework symmetry: $\sigma$ - symmetric in-plane reflection, 
$\pi$ - asymmetric out-of-plane reflection. Excited antibonding orbitals are $\sigma^{*}, \pi^{*}$, and $R$ (rydberg, principle quantum member excitation). In addition, following Lewis, we recognize "lone-pair" orbitals, $n$-orbitals as non-bonding, and $\ell$-orbitals as conjugation-capable (to a $\pi$-system) (see Fig. 8).

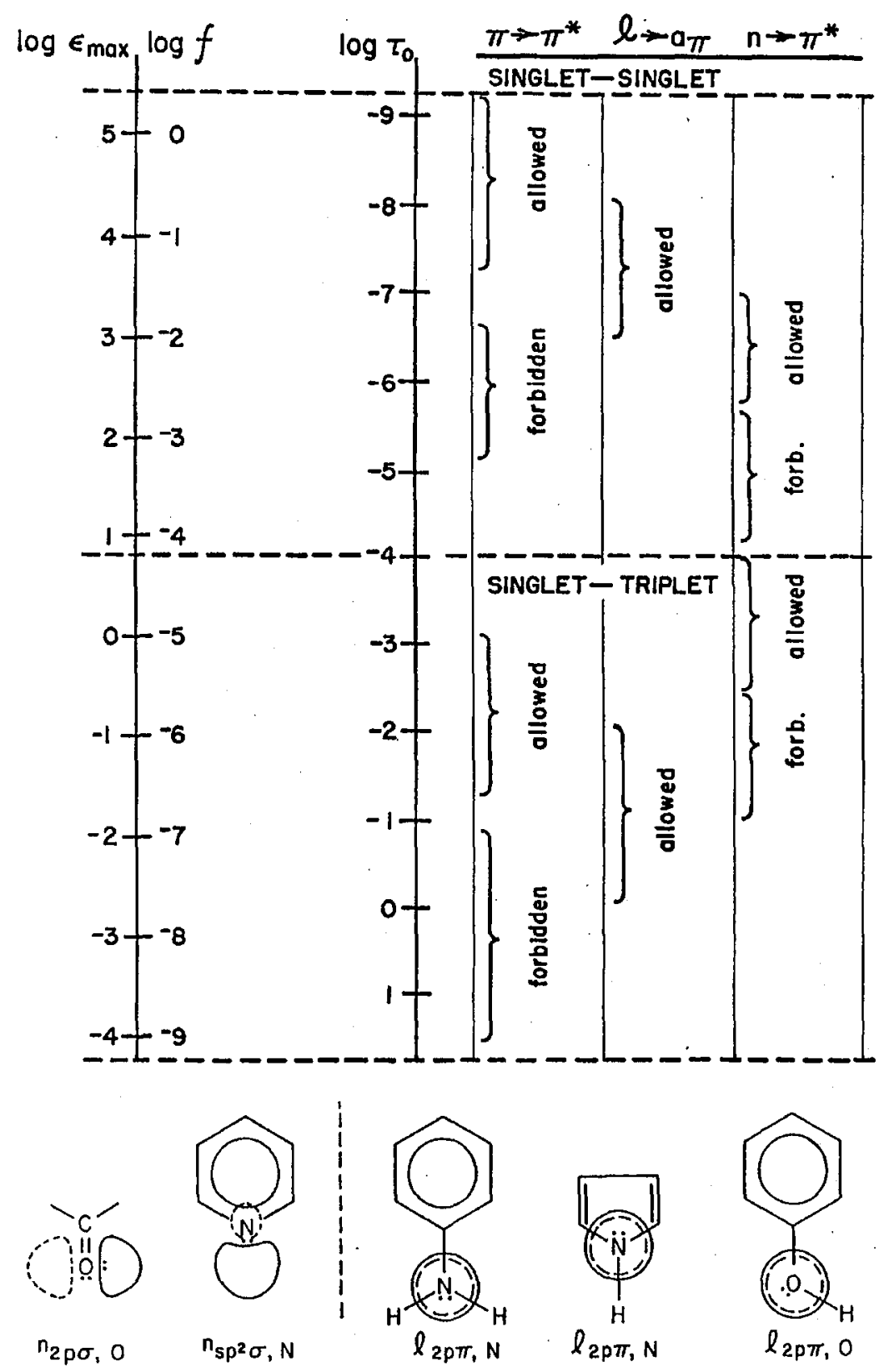

Fig. 8. Molecular electronic transitions $f$-number scale. 
Electronic transition types could then include $[15,16,21] \sigma \rightarrow \sigma^{*}, \pi \rightarrow \pi^{*}$, $\sigma \rightarrow \pi^{*}, \pi \rightarrow R, n \rightarrow \pi^{*}, \ell \rightarrow \pi^{*}, n \rightarrow \sigma^{*}$, etc.

Multiplicity classifications commonly may include singlet $S$, triplet $T$; doublet $D$, quartet $Q$. There are understood to be used in zeroth order for nomenclature purposes, although pure multiplicity states do not exist in reality: For very high $Z$, the designations lose meaning and are replaced.

Finally, in addition we recognize a series of types of charge transfer transitions, intramolecular and intermolecular, and also charge-translocation transitions, as proposed for ethoxyvinylpyrillium ion $[22,23]$.

The correlation chart [24] for molecular electronic transitions (Fig. 8) exhibits an interesting anomaly. The upper limit to transition probability of the various orbital types of transitions descends as one compares $\pi \rightarrow \pi^{*}, \ell \rightarrow a_{\pi^{*}}$, $n \rightarrow \pi^{*}$ singlet-singlet transitions. This trend has a direct explanation in the spatial overlap of the corresponding orbitals in the Cartesian framework.

The anomaly comes in the trends of triplet-singlet transitions. Recalling the singlet-triplet mixing result of Sec. 5, one would expect the fall of transition probability of the $\pi \rightarrow \pi^{*} \ldots n \rightarrow \pi^{*}$ triplet-singlet series to be paralleled in the singlet-singlet series. In reality, the correlation is seen to be reversed, or at least irregular. The resolution of this puzzle is to be found in the effect of the spin-orbital operator on the orbital orientation in the Cartesian framework, an effect unique to the world of polyatomic molecules.

The role of the angular momentum operator on orbital rotation [25] in spin-orbital coupling is shown in Fig. 9. In this figure the transition moment integral is displayed graphically for the effect of the angular momentum operator on the orbital transition of the three types $n \rightarrow \pi^{*}, \ell \rightarrow a_{\pi}$ (i.e., $\ell \rightarrow \pi^{*}$ ) and $\pi \rightarrow \pi^{*}$. In the spin-orbital Hamiltonian the components of the orbital angular momentum (vector) operator when analyzed can be shown to act like a rotation operator, e.g., $\widehat{M}_{y} \sim R_{y}$ (angular momentum about $y$ acting as a rotation about $y$ ), an $x$-directed orbital goes into $z$; and a $z$-orbital goes into $x$. Thus, in the $n \rightarrow \pi^{*}$ case (top of Fig. 9), the $n$-orbital $\left(s p^{2}\right)$ has a $p$-component along $z$, which upon rotation about the $y$-axis is directed along $x$ (shown as an $s p^{2} n$-orbital). As a result, the $n$-orbital overlap with the $\pi^{*}$-orbital at the $\mathrm{N}$-atom (of pyridine) would have maximum overlap as the one-center integral. Thus, an enhanced $T \rightarrow S$ emission probability would be expected.

In contrast, if we perform the analogous operation on the $\pi \rightarrow \pi^{*}$ case (bottom of Fig. 9), for the aniline molecule, rotating the C-atom $\pi$-orbital about the $z$-axis in this case, makes the overlap 0 even for the 2-center integral. As McClure showed in $\pi \rightarrow \pi^{*}$ transitions in planar aromatics, only 3-center integrals have non-zero value [26; cf. 27]. Thus, $T \rightarrow S$ transitions of $\pi \rightarrow \pi^{*}$ type in planar aromatics are especially restricted.

The $\ell \rightarrow \pi^{*}$ case of orbital promotion (Fig. 9) falls in the intermediate range in terms of spatial overlap and expected $T_{1} \rightarrow S_{0}$ probability.

All of the cases considered in the transition probability correlation diagram of Fig. 8 cover the polyatomic molecules consisting only of light-(low- $Z$ )-atoms. We now face the puzzle of how such molecules can have readily populated triplet 


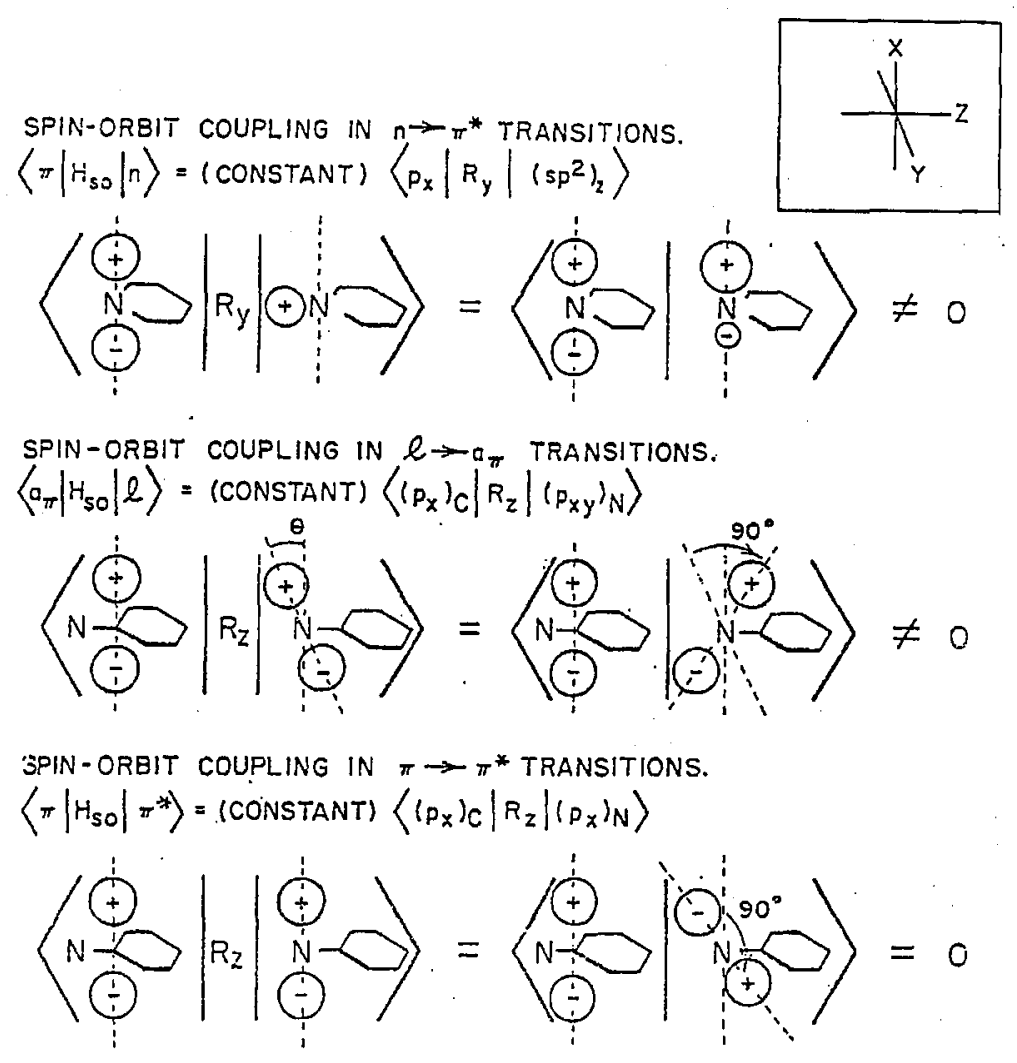

Fig. 9. Angular momentum operator: orbital rotation in spin-orbital coupling.

states, with quantum yields of $T_{1} \rightarrow S_{0}$ emission (phosphorescence) even as high as unity (zero fluorescence). The problem lies in the dynamics of polyatomic molecule excitation, and not in any revolutionary change in the quantum mechanical restrictions.

\section{The role of dynamics in triplet-state excitation}

The simultaneous total emission [16] of fluorescence and phosphorescence in organic molecules, exemplified by the case of phenanthrene (Fig. 3), illustrates a remarkable fact that the efficiency of triplet state excitation can be very high, even for a molecule made up of only $\mathrm{C}$ and $\mathrm{H}$ atoms. In addition, the singlet-triplet splits for ordinary organic molecules are observed to be [12] in the range of $5000-12000 \mathrm{~cm}^{-1}$ (cf. 2000 for dye molecules). The relatively rare occurrence of fluorescence in organic molecules in general is contrasted commonly by the appearance of strong phosphorescence. In fact, in molecules substituted with carbonyl, quinone, nitro, nitroso, and other unsaturated groups; and aza- $\mathrm{N}$ molecules such as pyridine, diazines, etc.; the typical characteristic is that phosphorescence emission is the highly dominant or unique luminescence property $[15,16]$, in spite of the presence of only $\mathrm{C}, \mathrm{N}, \mathrm{O}$, and $\mathrm{H}$ atoms. Figure 10 illus- 


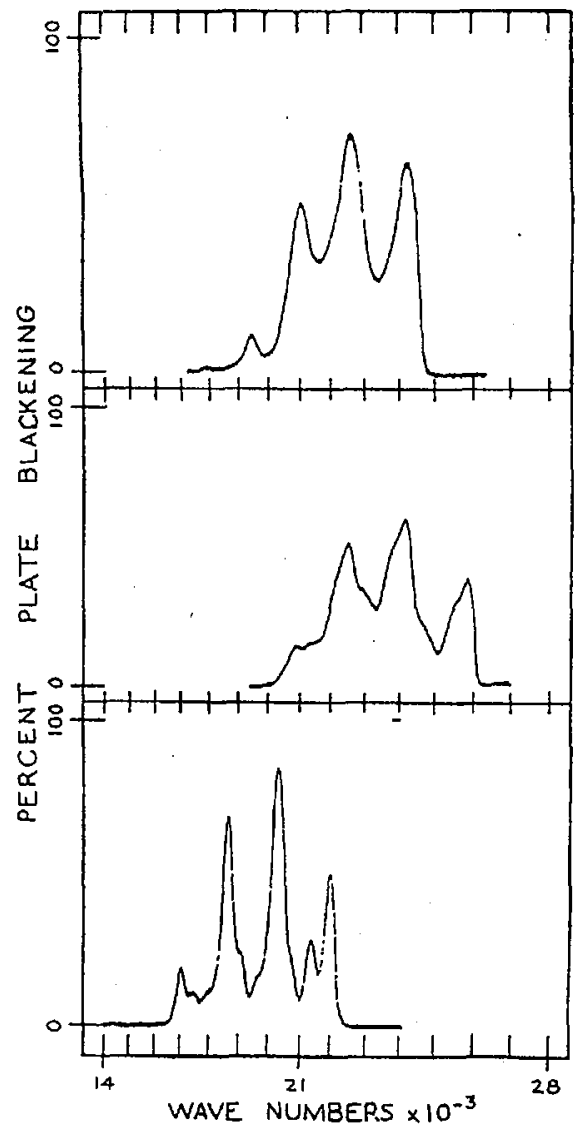

BENZOPHENONE

ACETOPHENONE

ANTHRAQUINONE

Fig. 10. Unique $T_{1} \rightarrow S_{0}$ luminescences in carbonyl compounds.

trates the $T \rightarrow S$ emission as the unique luminescence of carbonyl compounds, with quantum yield in some cases approaching unity.

We may make the statement that spin-orbital coupling, with its relativistic component, commonly dominates the molecular excitation dynamics of light-(low-Z)-atom molecules.

The early puzzle was, how can such a forbidden process as $T_{1} \rightarrow S_{0}$ emission, or $S_{1} \leadsto T_{1}$ excitation occur if the spin-orbital prohibition factor is of the order of magnitude of $10^{-6}$ on the rate constant? The answer lay not in an abnormal quantum mechanical enhancement effect, but in the dynamics of excitation. Physicists steeped in the behavior of excited states in atoms and diatomic molecules had not recognized the ultrafast radiationless transitions occurring in internal conversion rates in the $10^{12} \mathrm{sec}^{-1}$ range. Even with a prohibition factor of $10^{-6}$, an intersystem crossing $\left(S_{1} \leadsto T_{1}\right.$, Fig. 4) rate in the range $10^{6} \mathrm{sec}^{-1}$ could occur. Such a rate of radiationless transition, while not competing strongly with the fluorescence rate $\left(S_{1} \rightarrow S_{0}\right)$ in dye molecules, becomes very competitive if the $S_{1}$ state is of $\left(n, \pi^{*}\right)$-orbital configuration character, with a limited oscilla- 
tor strength (poor spatial overlap for the transition moment), and the enhanced $T \leftrightarrow S$ transition probability as discussed qualitatively in Sec. 6 . This enhancement was analyzed formally by Clementi and Kasha [28] for pyridine by group theoretical methods, and for pyridine and diazines by Goodman and Krishna [29A] by integral evaluation, showing definitively that the singlet-triplet intensity is derived from $T_{1}\left(n, \pi^{*}\right)-S_{1}\left(\pi, \pi^{*}\right)$ mixing. Goodman and Laurenzi presented the rigorous derivation of singlet-triplet transition probabilities with the essential relativistic quantum mechanical basis [29B]. Most often, the lowest singlet-triplet separation is the singlet-triplet split (the same $n, \pi^{*}$ configuration). Mostafa El-Sayed [30] applied the enhancement idea to intersystem crossing, especially in the case of $S_{n, \pi^{*}} \leadsto T_{\pi, \pi^{*}}$ for an analogous enhancement.

In summary, analysis [15] of the competitive excitation kinetics indicated clearly that the intersystem crossing rate $S_{1} \leadsto T_{1}$ (Fig. 4) could be comparable to the radiative rate $S_{1} \rightarrow S_{0}$, and often can be so dominant as to result in a unique $T_{1} \rightarrow S_{0}$ phosphorescence emission. Expressed otherwise, the Einstein coefficients $A_{21}$ and $B_{12}$ for the respective $T_{1} \rightarrow S_{0}$ emission and $S_{0} \rightarrow T_{1}$ absorption are limited by the appropriate spin-orbital perturbation according to normal quantum mechanical ranges. On the other hand, a high quantum yield of triplet state population, with its consequent dominant $T_{1} \rightarrow S_{0}$ luminescence, is the result of dynamic factors arising from typical ultrarapid polyatomic radiationless processes. In brief, a high quantum yield of $T_{1} \rightarrow S_{0}$ phosphorescence emission does not mean a high transition probability for this radiative process, but merely a rate for the spin-orbitally restricted $S_{1} \leadsto T_{1}$ radiationless process which competes favorably with the $S_{1} \rightarrow S_{0}$ radiative rate.

\section{Internal conversion and intersystem crossing}

The clear message of the preceding discussion is that the heart of the matter in the dynamics of molecular photophysical processes lies in understanding the nature of the radiationless mechanism of internal conversion and intersystem crossing.

The term internal conversion had been used by Perrin in 1929 [5], by Franck and Livingston in 1941 [6], and by other early researchers in molecular photophysics. Working with dye molecules focussed attention on the relation of the first principal absorption band in relation to the fluorescence emission. The use of the term internal conversion was confined mainly to what is now called intramolecular vibrational relaxation (IVR) within the first absorption band.

In 1941 Oppenheimer introduced internal conversion as derived from the nuclear physics phenomenon [31], applying the term to the unusual case of a guest dye molecule excitation transfer to a host photosynthetic system.

Internal conversion defined as the ultrarapid radiationless transitions between the excited electronic states of a like multiplicity appears to have been introduced by the author in $1947[12,15]$. Spectroscopic investigation of excitation of electronic absorption bands $S_{0} \rightarrow S_{n}$ above the first electronic absorption region $S_{0} \rightarrow S_{1}$, even for extremely high $S_{1} \rightarrow S_{0}$ fluorescence emission intensity, indicated that only the lowest excited state emission $S_{1} \rightarrow S_{0}$ was observable. The limits of $S_{n} \rightarrow S_{0}$ vs. $S_{1} \rightarrow S_{0}$ possible intensity ratios were set at approximately 1 to 10000 . 
These observations, carried out on a series of molecules, led to the two succinct statements [15] defining internal conversion and its consequences:

(a) The emitting electronic level of a given multiplicity is the lowest excited level of that multiplicity.

(b) The non-emitting upper electronic states will exhibit uncertainty broadening in $S_{0} \rightarrow S_{n}$ absorptions as a consequence of the shortened state lifetimes.

The first of these has been verified abundantly, and it is now recognized that exceptions are exceedingly rare. It should be noted that the advent of the femtosecond laser now turns a spotlight on real and measured rate constants for various cases. A detailed study of exact internal conversion pathways in excitation of aromatic hydrocarbons was also made [32].

The second rule (b) has been subjected to an elegant and extensive testing by Byrne and Ross [33] in their study of diffuseness in electronic spectra. They gave objective spectroscopic criteria for diffuseness, then tested these by seeking to observe intrinsic diffuseness in vapor spectra taken at moderately high resolving powers. These authors verified the generalization made by (b), again with a few exceptions. As an indication of the complexity of the spectroscopy of polyatomic molecules, Hochstrasser and Marzzacco [34] demonstrated intrinsic spectral diffuseness arising from embedded states.

Intersystem crossing as a spin-orbitally restricted internal conversion was introduced by the author $[12,15]$ in realization that lowest triplet states were excited by a radiationless mechanism. In this case also ultrafast lasers have a role in defining the mechanism of excitation in specific cases. Not only is this of interest in the case of aromatic hydrocarbons, in which there is an observable competition between $S_{1}$ and $T_{1}$ state excitations [16,32], but the case of $\mathrm{N}$-heteromatic molecules poses additional complexities in competitive excitation dynamics [35].

The importance of radiationless transitions in polyatomic molecules has produced a wealth of new researches on the topic.

Theoretical studies of radiationless transitions in polyatomic molecules have been very intensive and extensive. Among key topics investigated we may list:

- Gouterman's theory [36] of a solute molecule embedded in crystalline matrix, and coupled with the phonon field of the matrix, calculating the radiationless transition probability by analogy with radiation theory.

- Robinson and Frosch's theory [37] of solvent acting as an energy sink, formulated for radiationless transitions slow in comparison with vibrationless relaxation times.

- Coulson and Zalewskii's theory [38] generalized Zerner's method [39] for crossing of molecular potential functions, deriving the non-adiabatic transition probability.

- Ross and co-workers theory [32] of solely intramolecular radiationless transitions, focussing on tunneling phenomena and Franck-Condon factors [35].

- Siebrand's study [40] of Franck-Condon factors in the Robinson-Frosch theory.

- Lin's theory [41] is based on the deviations from the Born-Oppenheimer approximation, using the non-stationary aspects of the approximation. 
All of these theories have been reviewed critically [42]. One positive result of all of the above is the important theoretical deduction [37] of a very strong deuterium isotope effect on the $S_{1} \leadsto S_{0}$ and $T_{1} \leadsto S_{0}$ radiationless pathways. These predictions, important in the molecular photophysics of excited-state spectroscopy and photochemistry, have been applied frequently since their first demonstration [43].

One negative aspect in several cases of stated theories [32, 35, 37, 40, 41] is the assumption that vibrational relaxation is rapid compared with radiationless transition rates. Thus, the slower processes, e.g., $S_{1} \sim S_{0}$ and $S_{1} \leadsto T_{1}$ and $T_{1} \leadsto S_{0}$ are covered by their theories, but not $S_{n} \leadsto S_{1}$ or $T_{n} \leadsto T_{1}$. But these are the very rapid internal conversions of special interest in molecular photophysics. Even with the femtosecond and picosecond lasers now being available, considerable ingenuity will be required to devise experiments to measure such radiationless rates as $S_{3} \leadsto S_{2}, S_{3} \leadsto S_{3}^{\prime}$ (in excited state intramolecular proton-transfer (ESIPT)), $T_{1}^{\prime} \leadsto T_{1}$, and others. Nevertheless, these are important pathways in molecular photophysics.

Other negative aspects include the implication that the internal conversion (IC) process necessarily involves solute-solvent interaction $[36,37]$, and a failure to distinguish [38] between the behavior of small polyatomic molecules and larger polyatomics for which the IC process is ultrafast and has essentially a probability of 1 .

This last difficulty was elucidated in the work of Jortner and Berry [44], who classified polyatomic molecules by two distinct categories: those small molecules with discrete vibrational level interaction in excited states (resonance limit), and those large polyatomic molecules possessing such a high density of vibronic levels (of $S_{1}$ ), e.g., at the $S_{2}$ zero point level, as to represent a quasi-continuum (statistical limit).

Jortner and his colleagues [44-47] produced an avalanche of research on internal conversion. This work was summarized in two splendid reviews, with full literature citation $[48,49]$.

Especially influential has been the landmark paper of Bixon and Jortner [46], which showed that the quantum mechanical basis of the internal conversion lay in the failure of the Born-Oppenheimer approximation, and the necessity of including the kinetic energy operator for nuclear motion as the source of perturbation for IC to occur. The paper neatly introduces the known experimental facts recognized as observed for internal conversion (IC) and intersystem crossing (IS), and in conclusion presents a series of experimental predictions derived from the theory. A parallel study was made by Young [50].

The intervening period has led to a literature exploring many derivative refinements, including study of quantum beat phenomena, coherence dephasing, channel states, etc. Among the most interesting, from the point of view of femtosecond spectroscopy, is the study of uncertainty bandwidth of ultrafast excitation phenomena in relation to mechanism of energy coupling between the initially excited state and the receptor vibronic state. The contribution by Rhodes et al. [51] on the stationary approach in relation to radiation bandwidth was further amplified by Rhodes [52] and in the Perspective introduction and in the exploratory 
concluding section of the Henry and Kasha review [42]. The papers by Jortner and colleagues [44-47] have explored similar viewpoints on radiation bandwidth coupling effects. Among critical parameters brought out by theoretical researches is the role of the Franck-Condon factors in IC. It is recognized [42] that the Franck-Condon factors decrease inversely in magnitude as the density of vibronic states increases. Figure 11 illustrates the idea for perfectly nesting harmonic potentials.Even with some displacement of the minimum for large polyatomic molecule potentials, the Franck-Condon overlap decreases between the zero-point level of an $S_{n}$ state relative to the singlet state below it. This is in essence the origin of the band-gap-principle, which severely limits $S_{1} \leadsto S_{0}$ internal conversion.
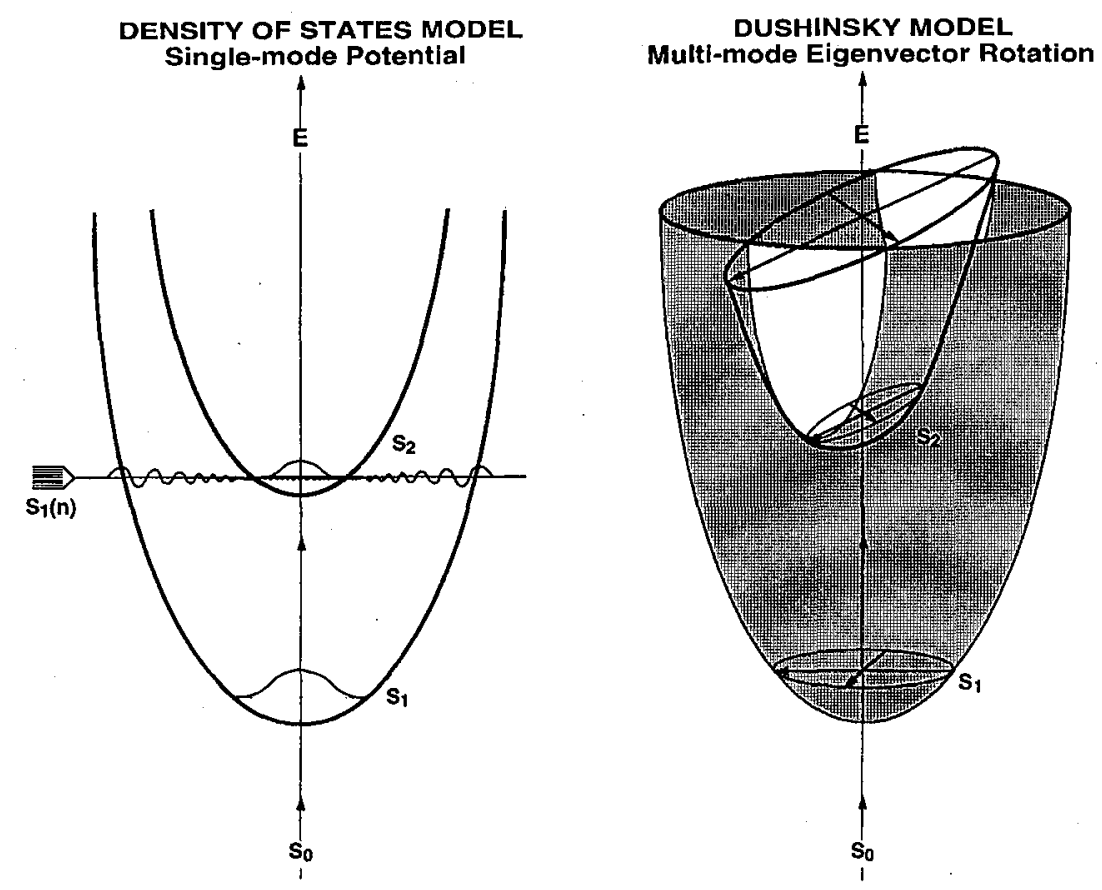

Fig. 11. Internal conversion models. Density of states model (single mode potential). Dushinsky model (multi-mode eigenvector rotation).

It is of interest here to consider the potential surfaces (hypersurfaces) suggested by the Dushinsky polyatomic Franck-Condon model [53] for simultaneous excitation of combinations of normal modes of vibration. The Dushinsky eigenvector rotation suggests that the overlap of two such potential surfaces for $S_{2}$ and $S_{1}$ and states may intersect at the corresponding maxima in the respective vibrational eigenfunctions. Such potential surface (or hypersurface) interactions would guarantee good overlap values of the Franck-Condon factor, offering another input to the distinctive feature of ultrarapid internal conversion in large polyatomics vs. diatomics and small polyatomics (e.g., having $3,4,5$ atoms). 
The enormous growth of the subject of radiationless transition theory and experiment is explored in the comprehensive recent book Radiationless Transitions in Polyatomic Molecules by Medvedev and Ocherov [54].

Femtosecond spectroscopy of internal conversion and intersystem crossing dynamic mechanisms offers an opportunity for fine-tuning of the theoretical predictions. Instead of assuming a range of rates for IC and IS steps, we would have available differentiation of rates determined by various experimental factors. The role of the solvent matrix, the specific orbital excitation types, the possible role of symmetry (if Herzberg-Teller-like matrix elements are involved), the role of excitation bandwidth as one probes the femto- to pico- to nano-second ranges, etc., each could introduce variations in measured IC and IS rates. This final stage will de-mystify these complex phenomena, and lead to refinements in the theory. With theory possibly well in hand, a whole new era of ultrafast dynamics promises to be developed.

\section{Photophysics of the triplet state}

The initial period of large polyatomic molecule triplet state studies required a succession of proofs that the controversial thesis that the phosphorescence of organic molecules in solid solution in glasses, commonly at $90 \mathrm{~K}$ or $77 \mathrm{~K}$, was really an electric-dipole triplet-singlet emission. The demonstration of intramolecular perturbation by high- $Z$-atom substitutions could be considered not perfectly exclusive of alternative solid state matrix phenomena observed for inorganic crystalline matrices.

The photomagnetism of the fluorescein dye (acid) form was determined in a posthumous paper [55] from the Lewis laboratory, based on a theoretical approach Lewis had indicated in scattered notes left in his office. A satisfactory value of magnetic moment for a triplet state was observed, verified by a more precise determination by Joussot-Dubien and Lesclaux [56]. Independent work by Fröhlich, Szalay and Ször [57] was extended later by Evans [58] to several molecules.

The electron spin-resonance proved to be elusive, and the absence of immediate results cast a negative cloud over the triplet-singlet assignment of phosphorescence. After a delayed interval, Hutchison and Mangum in 1958 [59], and van der Waals and de Groot in 1959 [60] succeeded in successfully observing the expected ESR splitting, in oriented molecules in an isomorphic crystal lattice, and in glass solvents, by these researchers respectively. This interval of 12 years added to the delay in the general acceptance of the triplet state assignmient.

Wide-angle interference patterns by the Selenyi method introduced by Weissman and Lipkin in 1942 [61] established that the electromagnetic multipole character of the fluorescein dye phosphorescence (acid form) was electric-dipole. Jabłoński thought that his metastable level could originate from a higher multipole or magnetic dipole, etc. Jabłoński's last doctoral student, Bissinger [62], extended Weissman's and Lipkin's work to a set of six organic molecules, including carbazole and triphenylamine, whose phosphorescence had bern well studied. His results showed that in all six cases, only electric-dipole transitions could account for the interference patterns observed. His Ph.D. dissertation [63] gives traces of the angular 
distributions of each interference pattern, the results excluding any other multipole. Jabłoński's colleagues report that Jabłoński did finally accept the triplet state assignment of the metastable state of organic molecules.

After the decade of uncertainty culminating in the demonstration of ESR spectroscopy of the lowest triplet state in organic molecules, a strong evolution of new triplet state studies ensued. This not only brought completeness to molecular studies of single-photon, single-molecule spectroscopy, but also provided key elements to the evolution of molecular photochemistry [64]. The Beirut conference on triplet states appeared in 1967 [65], and a first comprehensive textbook arrived [66]. Critics vanished from the scene and the observations of triplet state transitions became commonplace. Numerous other source books feature triplet state researches.

Here are singled out several of the observational advances which have broadened the subject.

Triplet state phosphorescence in liquid solutions [67] is a keynote observation in terms of the initial preoccupation with solid-state concepts which initially retarded the full conception of phosphorescence as an intrinsic molecular state property.

Triplet-triplet absorption spectroscopy was recognized early [7] by the Lewis laboratory in Berkeley as a characteristic phenomenon. This was usually carried out under steady-state conditions in the solid glass matrix medium, in which a population of excited states sufficient for $T_{1} \rightarrow T_{n}$ absorption could be recorded [68]. A major advance was made with the introduction of pulsed flash excitation which allowed spectroscopy of transient triplet state populations in liquid solvents to be investigated, starting with millisec to microsec timescales $[69,70]$.

Triplet-triplet energy transfer was discovered by Ermolaev and Terenin and recorded in a series of publications [71-73]. These studies have had a large impact in the development of organic photochemistry involving triplet-state "sensitization" of photochemical reactions [74].

Induced triplet-singlet transitions using high- $Z$-atom perturbations have taken their place as useful spectroscopic tools [18], becoming especially important for low-lying triplet states (e.g., $T_{1} \rightarrow S_{0}$ in infrared region) for which the radiationless intersystem crossing overcomes the $T_{1} \rightarrow S_{0}$ emission rate. Recently, Catalán [75] reported a major breakthrough on the observation of triplet-singlet transitions in proton-transfer spectroscopy, producing induced radiative transitions by a combination of intramolecular and intermolecular high- $Z$-atom perturbation. In spite of the large number of excited-state intramolecular proton-transfer cases, almost no $T_{1}^{\prime} \rightarrow S_{0}^{\prime}$ emissions had been observed previously for proton-transfer tautomers.

\section{Multi-molecule, multi-photon spectroscopy}

The subject of non-simple excitation modes covered by the title of this section - has arisen in the last three decades, largely as a result of the relative completeness of the concepts involved in simple excitation involving single-photon, one-molecule interaction. 
The list of these non-simple excitation cases is large and developing. Currently, we may list:

- Non-linear absorptions (two-photon spectroscopy) (higher terms in radiation field);

- Inter-molecular charge transfer transitions (CT);

- Twisting intramolecular charge transfer (TICT) (molecules with torsional modes);

- Dipolar relaxation spectroscopy (response of surroundings to dipole moment changes);

- Solvent cage spectroscopy (intermolecular perturbation of molecular potentials);

- Proton-transfer spectroscopy (ESIPT, ESDPT);

- Amplified spontaneous emission spectroscopy (ASE) (mirror-less lasing);

- Simultaneous transitions (two molecules, one photon; absorption, emission);

- Molecular exciton spectroscopy (coherent excitation; multi-molecule, one-photon);

- Unidirectional energy transfer (dissipative transfer in molecular pairs);

- Soliton spectroscopy (distortion migration upon excitation).

These subjects have extensive theoretical and experimental development and the range is too large to be developed here. However, it is clear that the numerous ultrafast steps in the dynamics of excitation of these systems call for the application of femtosec spectroscopy as an essential tool. One could then trace the course of evolution of the inter-molecular or intra-molecular excited state interaction required for the elucidation of the excitation dynamics. Especially interesting are anomalies in such phenomena, as exemplified by two recent cases which have appeared [76, 77]. It is just such anomalies which expand the wonderfully complex subject of polyatomic molecule electronic spectroscopy. The author plans to develop this section in a paper parallel to the present one.

\section{Epilogue}

The Aleksander Jabłoński Centennial presented the conferees of this meeting with the opportunity to reflect on the advances which the subject of polyatomic molecular spectroscopy has made. This development offers a historically vivid example of how clear thinking and concise formulation of an outline of a subject, however simple, extracted from very complex observations, can provide the solid foundation on which to build the magnificent edifice which now represents the subject of the electronic behavior of molecules in a photon field.

\section{References}

[1] A. Jabłoński, Nature 131, 839 (1933).

[2] A. Jabłoński, Z. Phys. 94, 38 (1935).

[3] M. Kasha, Acta Phys. Pol. A 71, 661 (1987).

[4] G.Q. Stokes, Philos. Trans. 142, 463 (1852); ibid. 143, 385 (1853).

[5] F. Perrin, Ann. Phys. (France) 12, 169 (1929). 
[6] J. Franck, R. Livingston, J. Chem. Phys. 9, 184 (1941).

[7] G.N. Lewis, D. Lipkin, T.T. Magel, J. Am. Chem. Soc. 63, 3005 (1941).

[8] A. Terenin, Acta Physicochim. URSS 18, 210 (1943) (English version).

[9] M. Kasha, J. Chem. Ed. 61, 204 (1984).

[10] H. Sponer, E. Teller, Rev. Mod. Phys. 13, 75 (1941).

[11] G.N. Lewis, M. Kasha, J. Am Chem. Soc. 66, 2100 (1944).

[12] M. Kasha, Chem. Rev. 41, 401 (1947).

[13] Jacob Bigeleisen, State University of New York, Stonybrook, private communication.

[14] G.N. Lewis, M. Kasha, J. Am. Chem. Soc. 67, 994 (1945).

[15] M. Kasha, Faraday Discuss. Chem. Soc. 9, 14 (1950).

[16] M. Kasha, Radiat. Res. 2, 243 (1960).

[17] D.S. McClure, J. Chem. Phys. 17, 905 (1949).

[18] M. Kasha, J. Chem. Phys. 20, 71 (1952).

[19] S.P. McGlynn, T. Azumi, M. Kasha, J. Chem. Phys. 40, 507 (1964).

[20] Y. Zeng, L. Biczek, H. Linschitz, J. Phys. Chem. 96, 5237 (1992).

[21] M. Kasha, in: Light and Life, Eds. W.D. McElroy, B. Glass, Johns Hopkins Press, Baltimore (MD) 1961, p. 31.

[22] M. Kasha, D. Parthenopoulos, B. Dellinger, J. Quantum Chem. 45, 689 (1993).

[23] V. Bonačić-Koutecký, Frei Universität, Berlin, private communication.

[24] M. Kasha, in: Proc. Int. Conf. on Lum., Vol. 1, Akad. Kiado, Budapest 1968, p. 166.

[25] M. Kasha, H.R. Rawls, Photochem. Photobiol. 7, 561 (1968).

[26] D.S. McClure, J. Chem. Phys. 20, 682 (1952).

[27] M. Mizushima, S. Koide, J. Chem. Phys. 20, 765 (1952).

[28] E. Clementi, M. Kasha, J. Mol. Spectrosc. 2, 297 (1958).

[29] (A) L. Goodman, V.G. Krishna, Rev. Ilod. Phys. 35, 541 (1963); (B) L. Goodman, B.J. Laurenzi, Advances Quantum Chemistry, Vol. 4, Academic Press, New York 1968, p. 153.

[30] M.A. El-Sayed, J. Chem. Phys. 38, 2834 (1963).

[31] J.R. Oppenheimer, Phys. Rev. 60, 158 (1941).

[32] G.R. Hunt, E.F. McCoy, I.G. Ross, Aust. J. Chem. 15, 591 (1962).

[33] J.P. Byrne, I.G. Ross, Aust. J. Chem. 24, 1107 (1971).

[34] R.M. Hochstrasser, C.J. Marzzacco, J. Chem. Phys. 48, 4079 (1968).

[35] J.P. Byrne, E.F. McCoy, I.G. Ross, A ust. J. Chem. 18, 1589 (1965).

[36] M. Gouterman, J. Chem. Phys. 36, 2846 (1962).

[37] G.W. Robinson, R.P. Frosch, J. Chem. Phys. 37, 1962 (1962); ibid. 38, 1187 (1963).

[38] C.A. Coulson, K. Zalewskii, Proc. R. Soc. Lond. A 268, 437 (1962).

[39] C. Zener, Proc. R. Soc. Lond. A 137, 696 (1932).

[40] W. Siebrand, J. Chem. Phys. 44, 4055 (1966); ibid. 46, 440 (1967). 
[41] S.H. Lin, J. Chem. Phys. 44, 3759 (1966).

[42] B.R. Henry, M. Kasha, Ann. Rev. Phys. Chem. 19, 161 (1968).

[43] M.R. Wright, R.P. Frosch, G.W. Robinson, J. Chem. Phys. 33, 934 (1968).

[44] J. Jortner, R.S. Berry, J. Chem. Phys. 48, 2757 (1968).

[45] D.P. Chock, J. Jortner, S.A. Rice, J. Chem. Phys. 49, 610 (1968).

[46] M. Bixon, J. Jortner, J. Chem. Phys. 48, 715 (1968).

[47] K.F. Freed, J. Jortner, J. Chem. Phys. 52, 6272 (1970).

[48] J. Jortner, S.A. Rice, R.M. Hochstrasser, Adv. Photochem. 7, 149 (1969).

[49] J. Jortner, J. Chim. Phys. 67, 1 (1970).

[50] J.H. Young, J. Chem. Phys. 51, 4061 (1969).

[51] W. Rhodes, B.R. Henry, M. Kasha, Proc. Nat. Acad. Sci., USA 63, 31 (1969).

[52] W. Rhodes, J. Chim. Phys. 67, 40 (1970).

[53] F. Dushinsky, Acta Physicochim., URSS 7, 551 (1937).

[54] E.S. Medvedev, V.I. Ocherov, Radiationless Transitions in Polyatomic Molecules, in Series in Chemical Physics, Vol. 57, Springer-Verlag, New York 1994.

[55] G.N. Lewis, M. Calvin, M. Kasha, J. Chem. Phys. 17, 804 (1949).

[56] J. Joussot-Dubien, R. Lesclaux, J. Chim. Phys. 61, 1631 (1964).

[57] P. Fröhlich, L. Szalay, P. Ször, Acta Chem. Phys. Univ. Szeged (Hungary) 2, 96 (1948).

[58] D.F. Evans, Nature 176, 777 (1955).

[59] C.A. Hutchison, Jr., B.W. Mangum, J. Chem. Phys. 29, 952 (1958).

[60] J.A. van der Waals, M.S. de Grost, Mol. Phys. 2, 333 (1959).

[61] S.I. Weissman, D. Lipkin, J. Am. Chem. Soc. 64, 1916 (1942).

[62] J. Bissinger, Bull. Acad. Pol. Sci. (Serie Math. Astron. Phys.) 17, No. 9, 597 (1969).

[63] J. Bissinger, Ph.D. Thesis, Institute of Physics, Nicholas Copernicus University, Toruń (Poland) 1970.

[64] M. Kasha, in: Comparative Effects of Radiation, Eds. M. Burton, J.S. Kirby-Smith, J.L. Magee, Wiley and Sons, New York 1960, p. 72.

[65] The Triplet State, Int. Sympos., Beirut (Lebanon), Ed. A.B. Zahlan, Cambridge University Press, Cambridge (U.K.) 1967.

[66] S.P. McGlynn, T. Azumi, M. Kinoshita, Molecular Spectroscopy of the Triplet State, Prentice-Hall, Englewood Cliffs (New Jersey) 1969.

[67] N.J. Turro, K.-C. Liu, M.-F. Chou, P. Lee, Photochem. Photobiol. 27, 523 (1978).

[68] D.S. McClure, J. Chem. Phys. 19, 670 (1951).

[69] G. Porter, M.W. Windsor, Faraday Discuss. Chem. Soc. 17, 178 (1954).

[70] G. Porter, F.J. Wright, Trans. Faraday Soc. 51, 1205 (1955).

[71] V.L. Ermolaev, A.N. Terenin, (S.I. Vavilov Memorial Volume), Akad. Nauk. SSSR 1952, p. 137.

[72] A.N. Terenin, V.L. Ermolaev, Izv. Akad. Nauk SSSR, Ser. Fiz, 26, 21 (1962).

[73] cf. V.L. Ermolaev, Sov. Phys.-Usp. 80, No. 1-2, 333 (1963). 
[74] G.S. Hammond, J. Saltiel, A.A. Lamola, N.J. Turro, J.S. Bradshaw, D.O. Cowan, R.C. Counsell, V. Vogt, C. Dalton, J. Am. Chem. Soc. 86, 3197 (1964).

[75] J. Catalán, C. Diaz, J. Phys. Chem. 102, 323 (1998).

[76] D. Gormin, A. Sytnik, M. Kasha, J. Phys. Chem. 101, 672 (1997).

[77] J.C. del Valle, M. Kasha, J. Catalán, J. Phys. Chem. 101, 3260 (1997). 